\title{
Directional Observation of Cold Dark Matter Particles (WIMP) in Light Target Experiments
}

\author{
Anna Anokhina ${ }^{1,2, *} \mathbb{C}$, Vasilisa Gulyaeva ${ }^{1}$, Emil Khalikov ${ }^{2}\left(\mathbb{D}\right.$, Evgeny Kurochkin ${ }^{1,2}$, Tatiana Roganova ${ }^{2}$, \\ Eduard Ursov $1,2, *(\mathbb{D}$ and Ivan Vidulin 1,2 (D) \\ 1 Department of Physics, Federal State Budget Educational Institution of Higher Education, \\ M.V. Lomonosov Moscow State University, 1(2), Leninskie Gory, GSP-1, 119991 Moscow, Russia; \\ guliaeva.vv19@physics.msu.ru (V.G.); evgeny0724648@yandex.ru (E.K.); vidulin.id18@physics.msu.ru (I.V.) \\ 2 Skobeltsyn Institute of Nuclear Physics (SINP MSU), Federal State Budget Educational Institution of Higher \\ Education, M.V. Lomonosov Moscow State University, 1(2), Leninskie Gory, GSP-1, 119991 Moscow, Russia; \\ nanti93@mail.ru (E.K.); rogatm@yandex.ru (T.R.) \\ * Correspondence: anokhannamsu@gmail.com (A.A.); ursovharleey@mail.ru (E.U.)
}

Citation: Anokhina, A.; Gulyaeva, V.; Khalikov, E.; Kurochkin, E.; Roganova, T.; Ursov, E.; Vidulin, I. Directional Observation of Cold Dark Matter Particles (WIMP) in Light Target Experiments. Universe 2021, 7, 215. https://doi.org/10.3390/ universe7070215

Academic Editors: Sunny Vagnozzi, Eleonora Di Valentino, Alessandro Melchiorri, Olga Mena and Luca Visinelli

Received: 7 May 2021

Accepted: 23 June 2021

Published: 28 June 2021

Publisher's Note: MDPI stays neutral with regard to jurisdictional claims in published maps and institutional affiliations.

Copyright: (C) 2021 by the authors. Licensee MDPI, Basel, Switzerland. This article is an open access article distributed under the terms and conditions of the Creative Commons Attribution (CC BY) license (https:// creativecommons.org/licenses/by/ $4.0 /)$.

\begin{abstract}
For the last 10 years, the search for dark matter (DM) was carried out taking into account the fact that the DM particles are WIMPs (Weakly Interacted Massive Particles) which were introduced in supersymmetric extensions of the Standard Model. Many experiments such as XENON1T, DarkSide, CRESST, etc. set the constraints on the WIMP-nucleon elastic interaction cross sections for different assumed WIMP masses. Methods for detecting WIMPs could play a special role, allowing one to determine the directions of the tracks of recoil nuclei and, therefore, to determine the preferred direction of the WIMP flux. In this work, we analyze the capabilities of such direct detection experiments through analyzing the lengths and directions of the tracks of recoil nuclei. Taking into account the existing experimental constraints, we conclude that the optimal target would be a lower density target containing nuclei of the $\mathrm{CNO}$ group, for example, liquid propane.
\end{abstract}

Keywords: WIMP; direct detection; nuclear recoils; light target; constraints

\section{Introduction}

The introduction of dark matter, along with modified theories of gravity (such as modified Newtonian dynamics (MoND) [1,2], entropic gravity [3] and quantised inertia [4]), is used to explain various astrophysical and cosmological phenomena such as distinctive shapes of the rotation curves of galaxies, dynamics of galaxy clusters, and effects of gravitational lensing and microlensing. However, nowadays, dark matter is a commonly accepted explanation of these phenomena, and there are various models to describe its interaction with baryonic matter.

WIMPs (Weakly Interacted Massive Particles) are well-known candidates for dark matter. It is assumed that our Galaxy is surrounded by a WIMP halo with WIMPs having masses ranging from $1 \mathrm{GeV}$ to $100 \mathrm{TeV}$. The idea of direct detection of WIMPs is based on the assumption that a wind of WIMPs with Maxwell velocity distributions with an average velocity of $220 \mathrm{~km} / \mathrm{s}$ "blows" on our Earth in the Solar system as it rotates around the center of the Galaxy [5]. In this scenario, WIMPs should be able to interact elastically with detector material producing recoil nuclei, which can be registered and measured. Some experiments are designed in such a way so as to allow for the determination of the recoil nuclei directions and, thus, the directions of WIMP momenta prior to their interaction with a target. The examples of such detectors are time-projection proportional chambers [6], micro pattern gaseous detectors [7], graphene-based detectors [8], and other detectors using advanced technologies.

The maximum of the assumed WIMP velocity distribution corresponds to the direction to the Cygnus constellation. Background events related to elastic neutrino interactions 
should be either isotropic (like the ones from both atmospheric and supernova neutrinos) or correlate with the position of the Sun (events from solar neutrinos). Consequently, the detection of excess events with directions pointing to the Cygnus constellation could serve as an unambiguous proof of the presence of dark matter in the form of a galactic halo [9].

In the present work, we provide the model constraints on the possibility of WIMP detection in a directional experiment with a light target, such as hydrogen nuclei $(\mathrm{H})$, nuclei of carbon-oxygen-nitrogen group (CNO), as well as fluorine nuclei (F). This work is organized as follows. In Section 2, we briefly describe a classical scheme of WIMP elastic interactions with recoil nuclei of the detector and provide the characteristics of WIMPs with different masses. In Section 3, we describe the scheme for modeling of recoil nuclei tracks in the detector material and study the features of tracks in nuclear emulsion, liquid propane $\mathrm{C}_{3} \mathrm{H}_{8}$, and liquid octafluoropropane $\mathrm{C}_{3} \mathrm{~F}_{8}$. Section 4 is devoted to numerical estimates of a possible WIMP signal obtained using the micrOMEGAs code [10]. Since solar neutrinos represent a directional background for WIMP search experiments, Section 5 considers recoil tracks from directional (solar) neutrinos and provides the main formulas for calculation of energy and angular distributions of recoil nuclei from neutrino elastic interactions with detector material nuclei, as well as the features of recoil nuclei tracks in nuclear emulsion, propane, and octafluoropropane. Finally, in Section 6 we discuss the possible number of recoil nuclei from WIMP and solar neutrino interactions with detector material, and draw conclusions concerning preferred targets for visualizing and directional WIMP experiments with WIMPs having the lowest supposed masses of 4-10 GeV.

\section{Scheme of WIMP Elastic Interaction with Detector Material. Energy and Angular Distributions of Recoil Nuclei}

Three models of tracking detectors with significantly different densities of sensitive materials have been considered. The first one is the NEWSdm nuclear emulsion detector [11] with density $\rho=3.1 \mathrm{~g} / \mathrm{cm}^{3}$, which uses nanoemulsion NIT (Nano Imaging Tracker) with grain sizes of 20-40-80 $\mathrm{nm}$ [12]. This is a huge improvement over a typical nuclear emulsion used, for example, in the OPERA $v_{\mu} \rightarrow v_{\tau}$ neutrino oscillation search experiment [13], where the emulsion grain size was approximately $200 \mathrm{~nm}$. The second model is that a detector in the form of $500 \mathrm{~L}$ of liquid propane $\mathrm{C}_{3} \mathrm{H}_{8}$ with density $\rho=0.495 \mathrm{~g} / \mathrm{cm}^{3}$, which works as a bubble chamber. As the third model we considered the sensitive material of the projected PICO experiment detector [14] - a bubble chamber filled with $500 \mathrm{~L}$ of octafluoropropane $\mathrm{C}_{3} \mathrm{~F}_{8}$ with density $\rho=1.601 \mathrm{~g} / \mathrm{cm}^{3}$.

The elastic interaction of WIMPs on target nuclei has been described in numerous works, e.g., $[15,16]$. Its general scheme is shown in Figure 1.

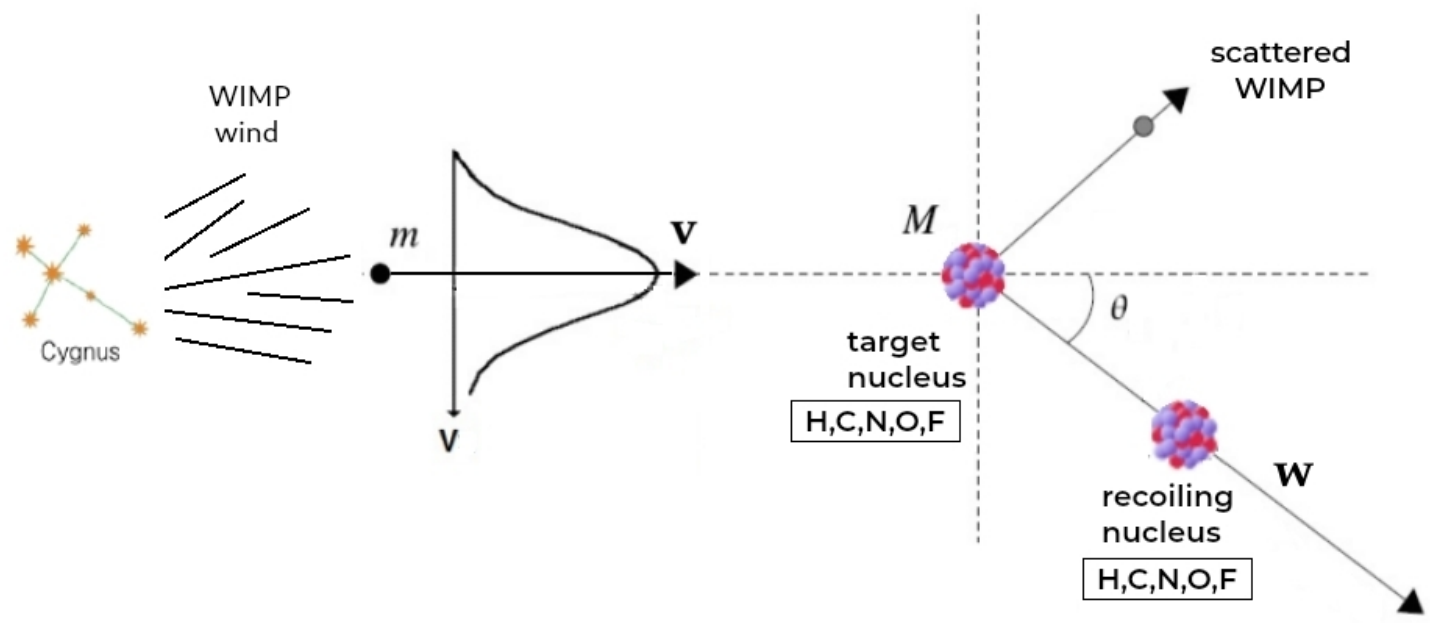

Figure 1. General scheme of WIMP elastic interaction with a target nucleus, in which a recoil nucleus is produced. WIMP velocities before the interaction follow a Maxwell distribution with an average velocity $220 \mathrm{~km} / \mathrm{s}$ and $\sigma=156 \mathrm{~km} / \mathrm{s}$. 
To model the tracks of recoil nuclei in the detectors of directional experiments one could use a double differential distribution of recoil nuclei by energy and direction described by the following formula [16]:

$$
\frac{d^{2} R}{d E d \Omega}=\frac{\rho}{4 \pi \mu^{2} m} \widehat{f}(w, \widehat{\mathbf{w}}) \sigma_{A}(E),
$$

where $d \Omega=d \phi d \cos \theta, \rho$ is the WIMP local density, $m$ is the WIMP mass, $M$ is the mass of the target nucleus, $\mu^{2}=m M /(m+M)$ is the reduced mass of the WIMP-nucleus system. $w=c \sqrt{M E / 2 \mu^{2}}$ is the minimal WIMP velocity necessary for the transfer of energy $E$ to a target with the mass $M$.

Just as it was done in [16], we take a modified Maxwell distribution as a $\widehat{f}(w, \widehat{\mathbf{w}})$ function:

$$
\begin{aligned}
& \widehat{f}(w, \widehat{\mathbf{w}})=\int \delta(\mathbf{v} \cdot \widehat{\mathbf{w}}-w) f(\mathbf{v}) d^{3} v, \\
& f(\mathbf{v})=\frac{1}{\left(2 \pi \sigma_{V}^{2}\right)^{3 / 2}} \exp \frac{-(\mathbf{v}-\mathbf{V})^{2}}{2 \sigma_{V}^{2}},
\end{aligned}
$$

where $\mathbf{v}$ is the WIMP velocity before the scattering, and $\widehat{\mathbf{w}}=\mathbf{w} /|\mathbf{w}|$ is the direction of recoil nucleus velocity $\mathbf{w}$ after the WIMP scattering on them. $\mathbf{w}$ and $\mathbf{v}$ are set with regard to the direction corresponding to the maximum of WIMP velocity distribution- $\mathbf{V}$.

The cross section of WIMP scattering on nuclei consists of two parts: spin-dependent (SD) and spin-independent (SI).

$$
\sigma_{A}(E)=\sigma_{A}^{S I}(E)+\sigma_{A}^{S D}(E) .
$$

Correspondingly, one can separate the spin-independent and spin-dependent contributions to the directional recoil rate.

The spin-independent part could be written as:

$$
\sigma_{A}^{S I}=\frac{4 \mu^{2}}{\pi}\left(\lambda_{p} Z+\lambda_{n}(A-Z)\right)^{2} F_{A}^{2}(q),
$$

where $\lambda_{p}, \lambda_{n}$ are amplitudes for DM scattering on nucleons; $Z$ and A are nucleus mass number and charge, respectively, and $F_{A}(E)$ is the Helm form-factor [17]:

$$
F_{A}^{H e l m}\left(q^{2}\right)=\frac{3 j_{1}\left(q r_{n}\right)}{\left(q r_{n}\right)} \exp \frac{-\left(q^{2} s^{2}\right)}{2},
$$

where $q$ is the momentum of the recoil nucleus, $r_{n}^{2}=c^{2}+\frac{7}{3} \pi^{2} a^{2}-5 s^{2}, s=1 \mathrm{fm}, c=$ $\left(1.23 A^{1 / 3}-0.6\right) \mathrm{fm}$,

$$
j_{1}\left(q r_{n}\right)=\frac{\sin \left(q r_{n}\right)}{\left(q^{2} r_{n}^{2}\right)}-\frac{\cos \left(q r_{n}\right)}{\left(q r_{n}\right)}
$$

The spin-dependent $\left(J_{A}\right.$ is the spin of the nucleus) part of the cross section $\sigma_{n}^{S D}(E)[18]$

$$
\sigma_{A}^{S D}=\frac{4 \mu_{A}^{2}}{\left(2 J_{A}+1\right)}\left(a_{p}^{2} S_{p p}(E)+a_{n}^{2} S_{n n}(E)+a_{p} a_{n} S_{p n}(E)\right)
$$

is defined through functions $S_{p p}(E), S_{n n}(E)$, and $S_{p n}(E)$, which play the same role as the nuclear form-factor $F\left(q^{2}\right)$ in the spin-independent case and are defined as [19]:

$$
S_{p p}(E)=S_{00}+S_{11}+S_{01}
$$




$$
\begin{gathered}
S_{n n}(E)=S_{00}+S_{11}-S_{01} \\
S_{p n}(E)=2\left(S_{00}+S_{11}\right),
\end{gathered}
$$

where $S_{00}, S_{11}$, and $S_{01}$ are the nuclear spin structure functions [19].

If the spin of the nucleus can be approximated by the spin of an odd nucleon only, then for a nucleus with an odd proton we have:

$$
S_{p p}=\frac{\lambda_{n}^{2} J_{A}\left(J_{A}+1\right)\left(2 J_{A}+1\right)}{\pi}, S_{n n}=0, S_{p n}=0,
$$

and for a nucleus with an odd neutron we get:

$$
S_{n n}=0, S_{n n}=\frac{\lambda_{n}^{2} J_{A}\left(J_{A}+1\right)\left(2 J_{A}+1\right)}{\pi}, S_{p n}=0 .
$$

Values of $\lambda_{n}^{2} J_{A}\left(J_{A}+1\right)$ were taken from $[15,20]$.

As targets we considered light $\mathrm{H}, \mathrm{C}, \mathrm{N}, \mathrm{O}$ nuclei, which make up the nuclear emulsion is the sensitive part of the NEWSdm detector [11], model propane bubble chamber, as well as fluorine $\mathrm{F}$, which is part of $\mathrm{C}_{3} \mathrm{~F}_{8}$ detector in the PICO experiment [14].

Hydrogen and fluorine nuclei have spins of $1 / 2$, while carbon and oxygen have zero spins. Another component of one of the targets considered by us is nitrogen, which has nuclei of a similar mass and a spin of 1 . However, unfortunately, we were not able to find any information on its $S_{01}$ structure function for an odd-odd nucleus, which is necessary for calculating the spin-dependent part, so for $\mathrm{N}$ we used a spin-independent approximation instead.

Formula (1) was used to calculate the two-dimensional probability density functions of energy $E$ and direction $\cos \theta$ distributions of various recoil nuclei. Then, in accordance to these distributions we simulated sets of individual recoil nuclei with fixed $E$ and $\cos \theta$ for WIMPs with masses 10, 60, 100, and $1000 \mathrm{GeV}$, see Figures 2-4.

The absolute values of WIMP-nucleon interaction cross-sections as well as the number of events per unit mass of the material have been obtained using the publicly available micrOMEGAs toolkit [10] for specific models of WIMPs.

Figures 2-4 show the examples of distributions of $10^{5}$ simulated hydrogen $(\mathrm{H})$, carbon $(\mathrm{C})$, and fluorine $(\mathrm{F})$ recoil nuclei over their energies $E_{\text {rec }}$ and the projection of a 3D recoil nucleus incidence angle on a 2D detector plane $\operatorname{tg} \theta_{z}$ for various WIMP masses. For the task of reconstructing the recoil nuclei track directions it is the projection of the $3 \mathrm{D}$ incidence angle $\theta$ on the detector plane (e.g., on the plane of an emulsion plate) that represents the angle corresponding to the track direction, which can be studied in the experiment, most accurately.

It is worth mentioning that there are noticeable differences in the energies of hydrogen, carbon and fluorine nuclei as well as almost indistinguishable shapes of angular distributions for $\mathrm{H}, \mathrm{C}$ and $\mathrm{F}$ for different WIMP masses. 

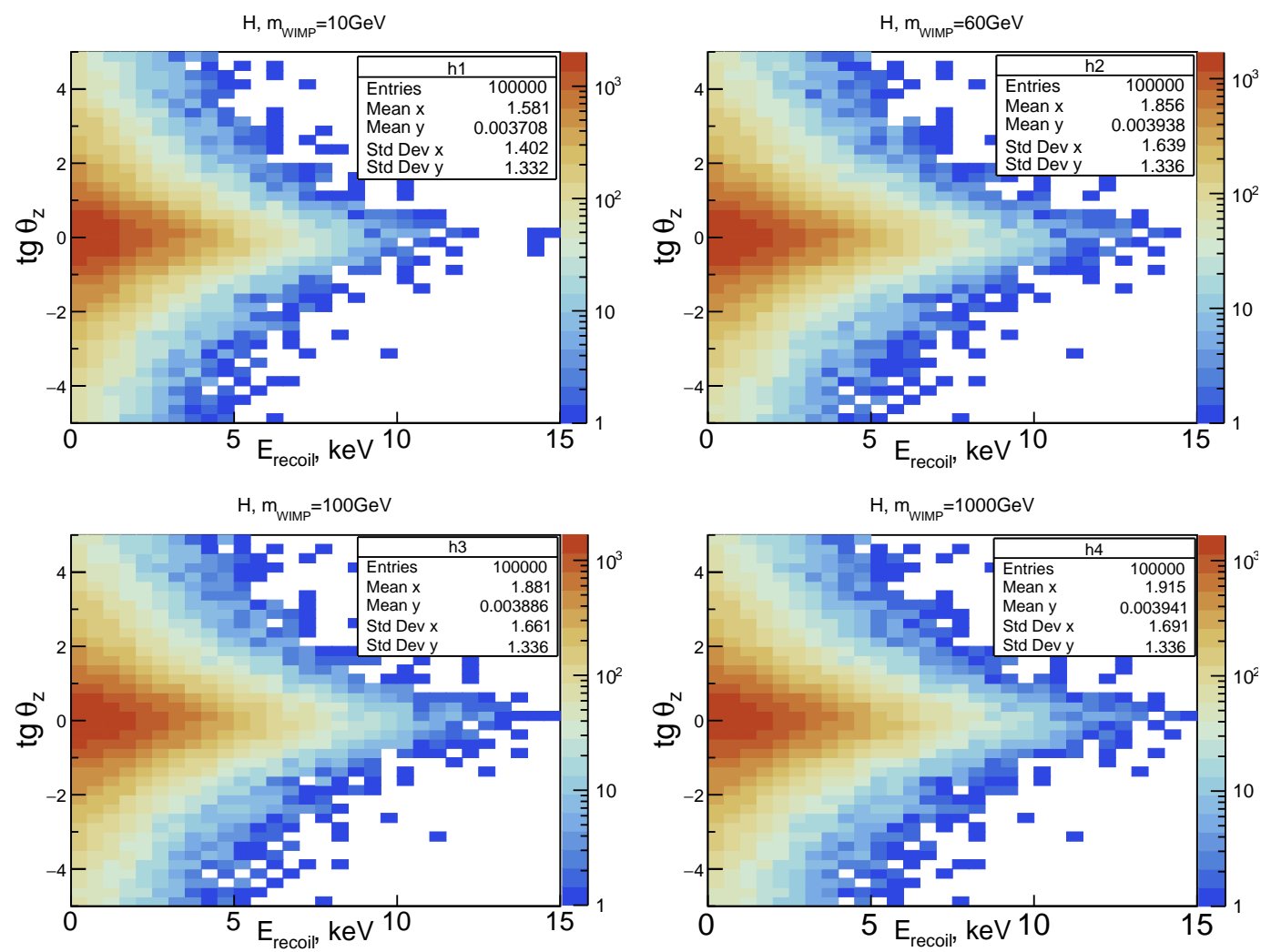

Figure 2. Distributions of $10^{5}$ simulated hydrogen $(\mathrm{H})$ recoil nuclei over their energies $E_{r e c}$ and the projection of a 3D recoil nucleus incidence angle on a $2 \mathrm{D}$ detector plane $\operatorname{tg} \theta_{z}$ for various WIMP masses.
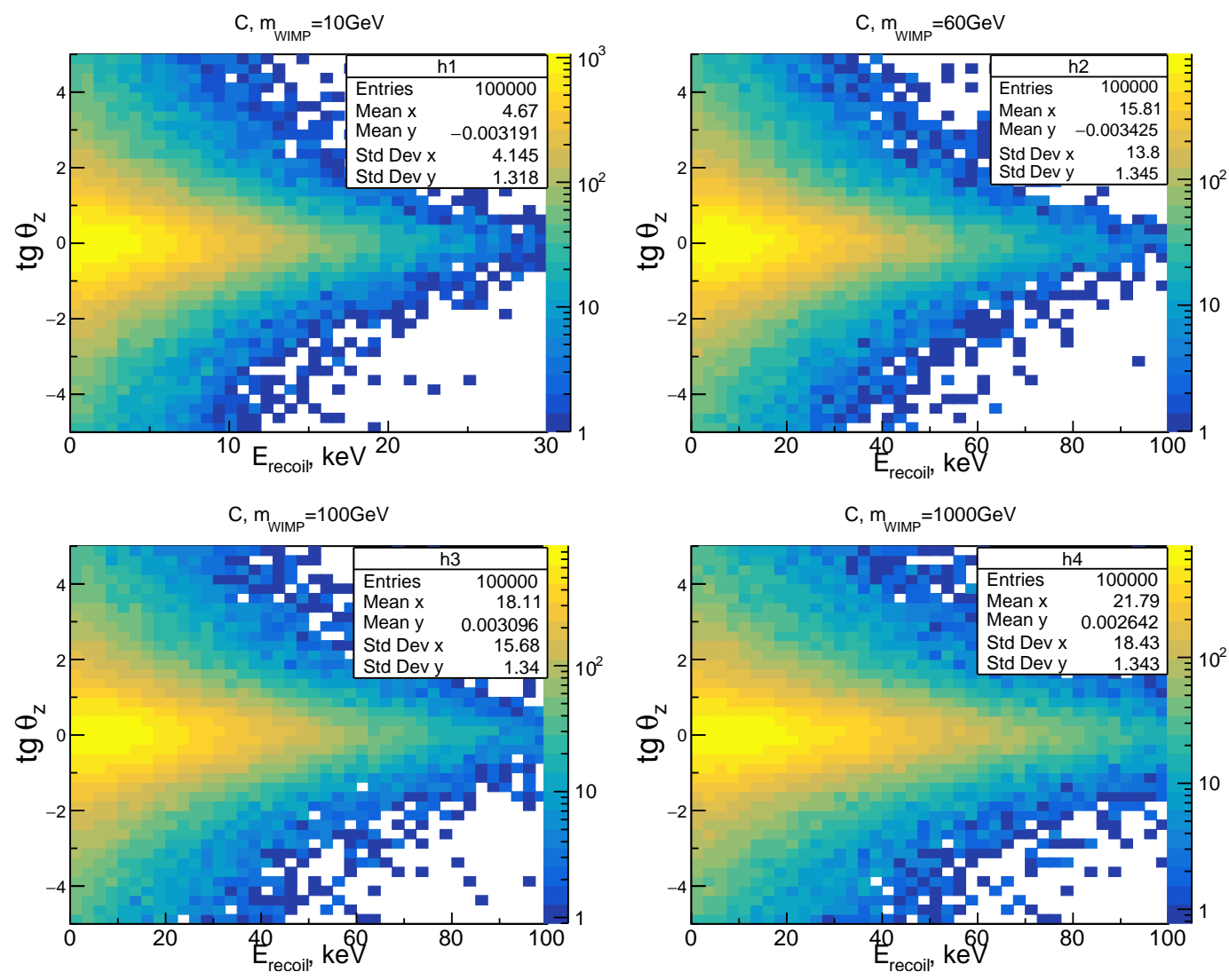

Figure 3. Distributions of $10^{5}$ simulated carbon $(C)$ recoil nuclei over their energies $E_{\text {rec }}$ and the projection of a $3 \mathrm{D}$ recoil nucleus incidence angle on a $2 \mathrm{D}$ detector plane $\operatorname{tg} \theta_{z}$ for various WIMP masses. 

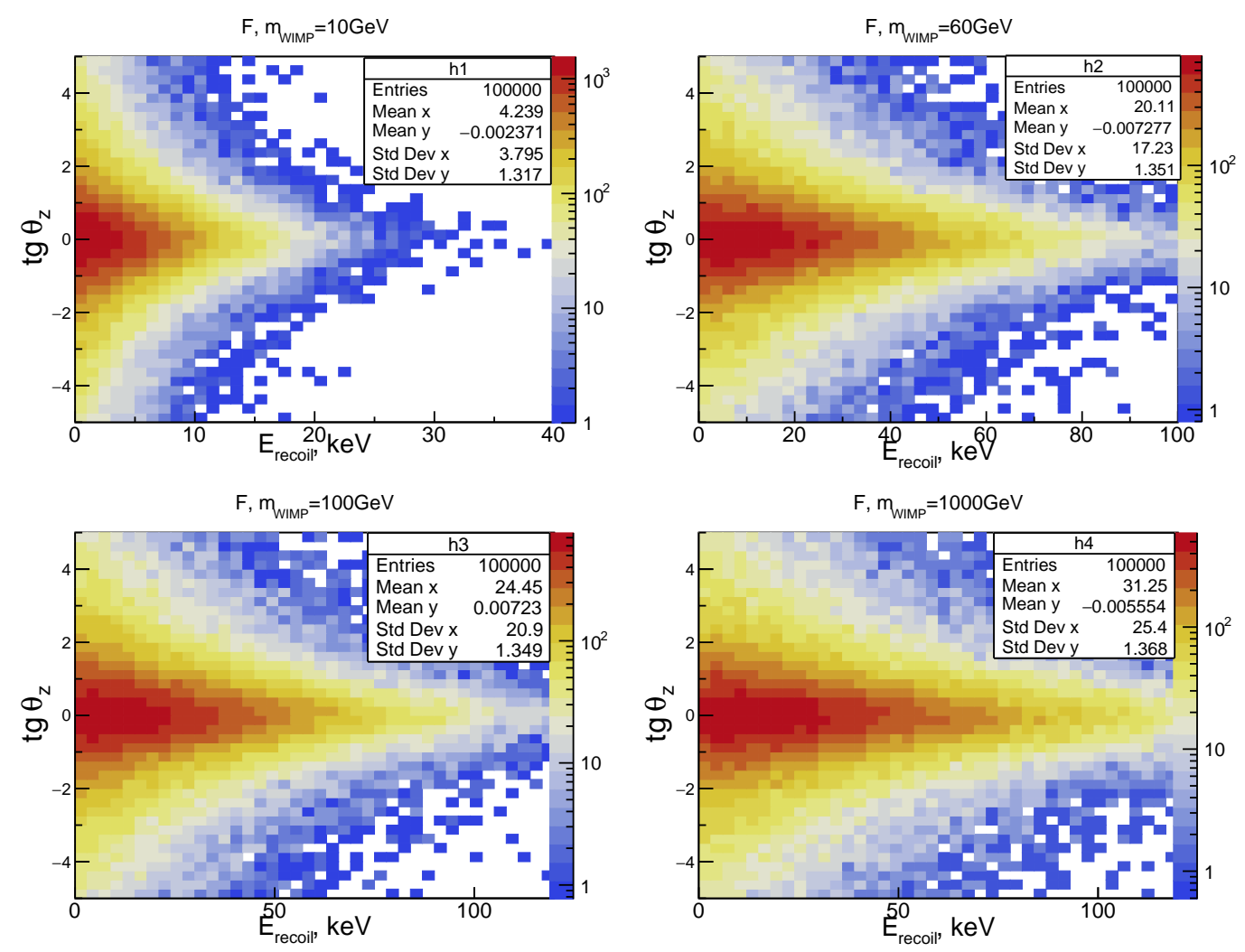

Figure 4. Distributions of $10^{5}$ simulated fluorine $(\mathrm{F})$ recoil nuclei over their energies $E_{r e c}$ and the projection of a 3D recoil nucleus incidence angle on a 2D detector plane $\operatorname{tg} \theta_{z}$ for various WIMP masses.

\section{Scheme of Track Modeling in GEANT4}

Modeling of the recoil nuclei tracks in the detector material has been done using GEANT4 toolkit. More specifically, we have used StandardNR-Nuclear Recoil Physics List) [21].

Examples of hydrogen and carbon nuclei tracks are shown in Figure 5. This figure also demonstrates the lines that represent best fits (according to the least squares method) of all the track points. Lengths and orientations of these tracks are then used to determine lengths and directions of recoil nuclei tracks.
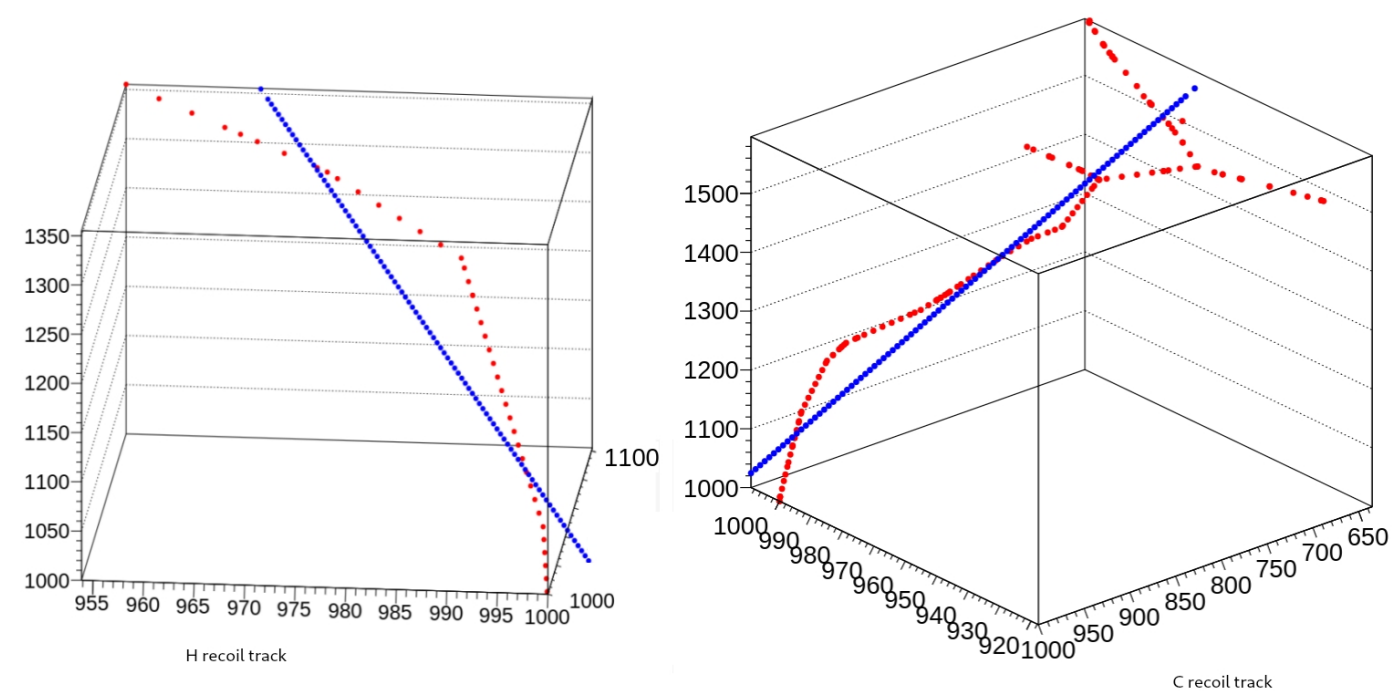

Figure 5. Examples of tracks of hydrogen (H) (left plot) and carbon (C) (right plot) nuclei in the nuclear emulsion obtained using the GEANT4 Nuclear Recoil Physics List. Red dots represent track points, the blue line is the least squares best fit of track points. The units are nm. 
The first step of our simulation included studying the tracks from monoenergetic hydrogen $\mathrm{H}(2,5,10,30 \mathrm{keV})$ and carbon $\mathrm{C}(10,30,60,100 \mathrm{keV})$ nuclei with fixed energies, which were emitted as a focused beam and interacted with nuclear emulsion material with density $3.1 \mathrm{~g} / \mathrm{cm}^{3}$. This modeling is necessary to estimate the extent of scattering of recoil nuclei and resulting track lengths of nuclei with various energies. The track lengths of carbon $(\mathrm{C})$ and hydrogen $(\mathrm{H})$ nuclei in the emulsion as well as angular distributions over the angle defined as an angle between the best fit of track points and the original direction of the recoil nucleus are shown in Figures 6 and 7 for various energies.
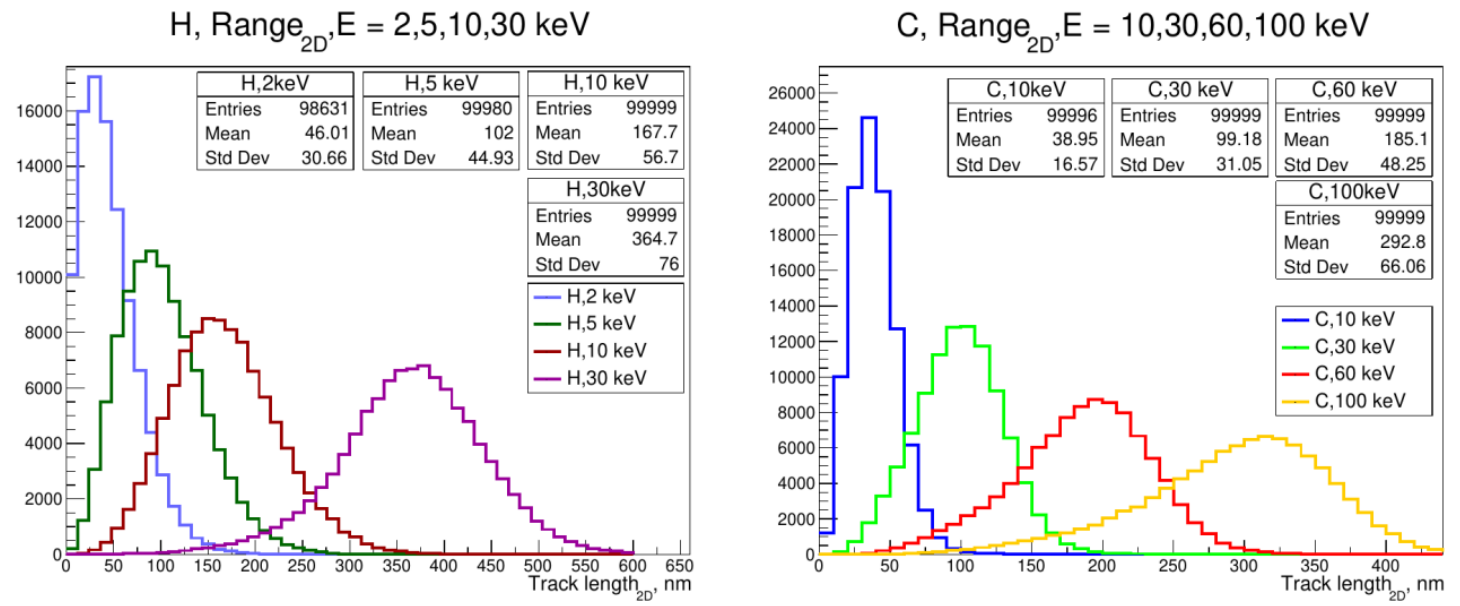

Figure 6. Distributions of model hydrogen $(\mathrm{H})(\mathbf{l e f t})$ and carbon $(\mathrm{C})$ (right) nuclei tracks over the lengths of their track lengths in the emulsion.

$\mathrm{H}, \theta_{2 \mathrm{D}}, \mathrm{E}=2,5,10,30 \mathrm{keV}$

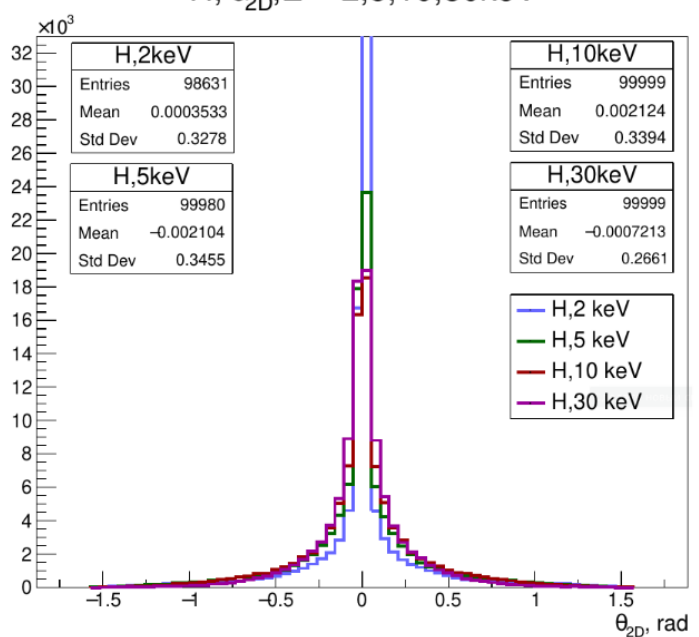

$\mathrm{C}, \theta_{2 \mathrm{D}}, \mathrm{E}=10,30,60,100 \mathrm{keV}$

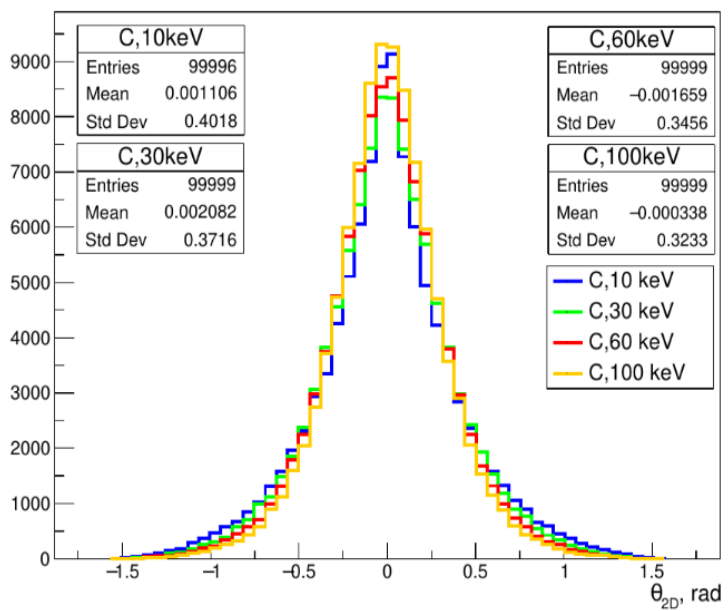

Figure 7. Distributions of model hydrogen $(\mathrm{H})$ and carbon $(\mathrm{C})$ nuclei tracks over the angle between the primary direction of a recoil nucleus and the direction obtained as best fit of their track points in the nuclear emulsion. These distributions determine the extent of scattering of recoil nuclei as they propagate through the emulsion material. The left figure shows the case for $(\mathrm{H})$, the right-for $(\mathrm{C})$.

Distributions of $10^{5}$ hydrogen $(\mathrm{H})$ and carbon $(\mathrm{C})$ nuclei recoil tracks in a nuclear emulsion over the tracks length and angular distributions relative to the direction "toward the Cygnus constellation" are shown in Figures 8 and 9 for WIMP masses 10 and $100 \mathrm{GeV}$. The tracks was modeled with GEANT4 StandardNR Physics List. The initial nuclear recoil energies $E_{\text {recoil }}$ was simulated based on the two-dimensional probability density functions (1) and shown in Figure 2 and 3. 
Only tracks with a length greater than $80 \mathrm{~nm}$ are considered.

As an alternative option for the detector material we considered liquid propane $\mathrm{C}_{3} \mathrm{H}_{8}$ with density $\rho=0.493 \mathrm{~g} / \mathrm{cm}^{3}$, which could be used as a tracker in the bubble chamber mode, and the target of the PICO detector [14] —octafluoropropane $\mathrm{C}_{3} \mathrm{~F}_{8}$ with density $\rho=1.601 \mathrm{~g} / \mathrm{cm}^{3}$. As for the case of the nuclear emulsion, we simulated $10^{5}$ recoil nuclei tracks from WIMPs with masses 10 and $100 \mathrm{GeV}$.

Distributions of track lengths in liquid propane for hydrogen and carbon recoil nuclei and WIMPs with masses 10 and $100 \mathrm{GeV}$ are shown in Figures 8 and 9, right.
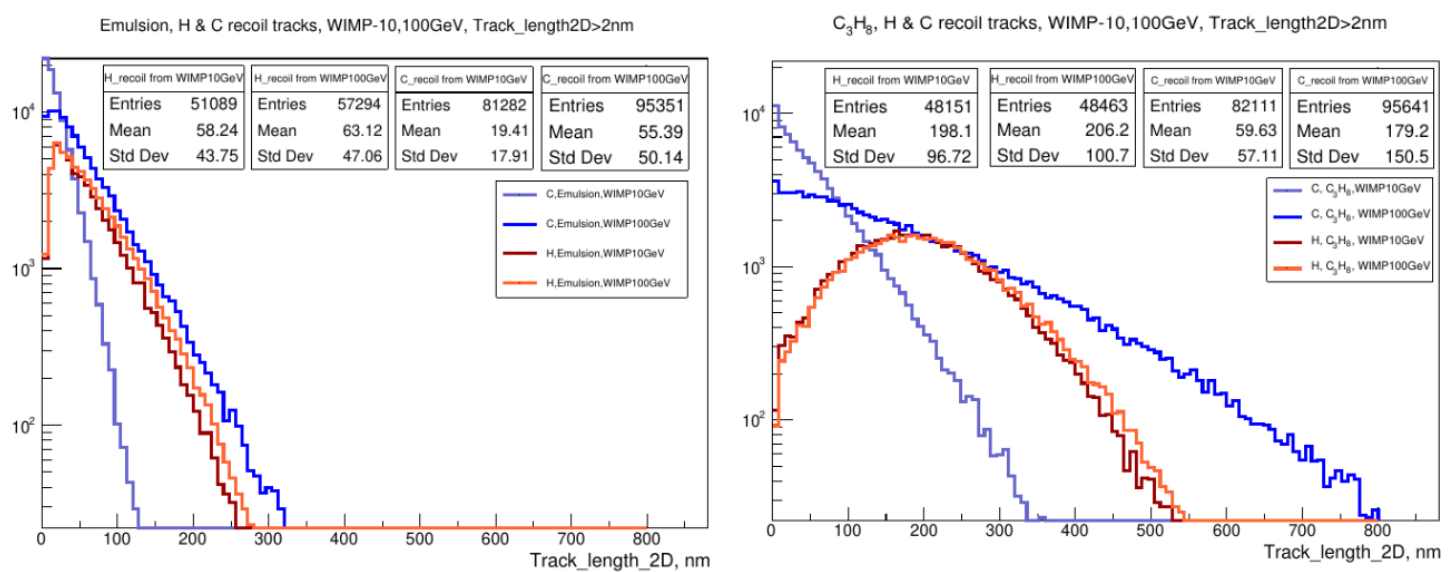

Figure 8. Distributions of track lengths in the nuclear emulsion (left) and the propane target (right) for hydrogen and carbon recoil nuclei for WIMPs with masses 10 and $100 \mathrm{GeV}$.
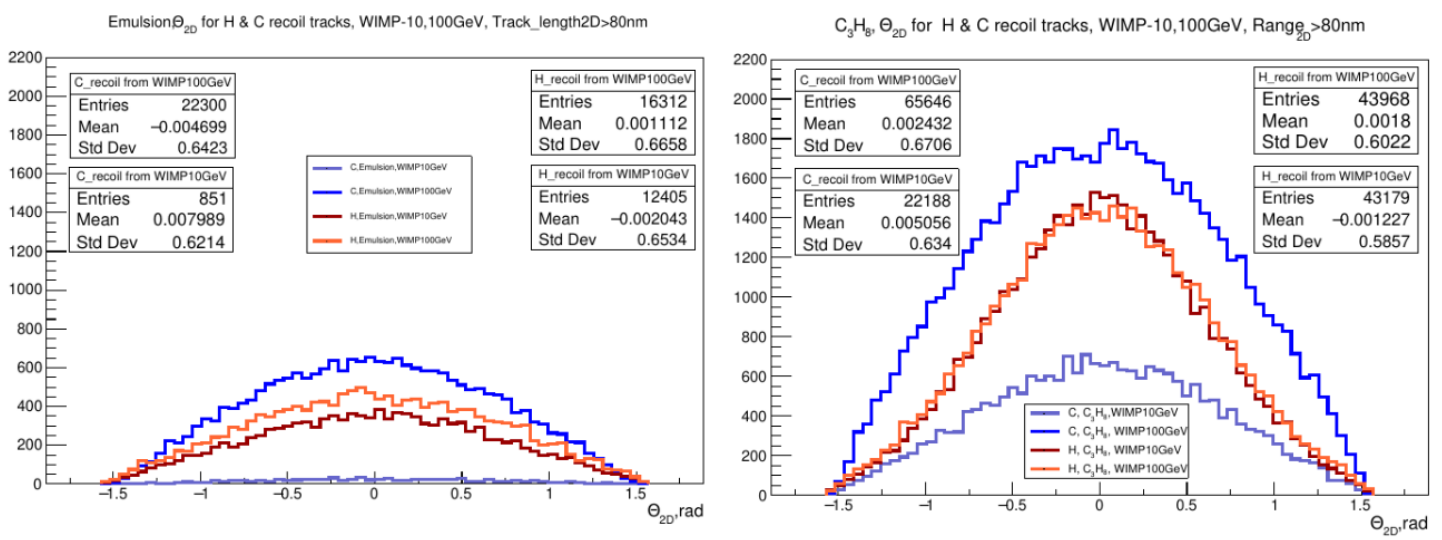

Figure 9. Angular distributions relative to the direction «toward the Cygnus constellation» of tracks in a nuclear emulsion (left) and in liquid propane (right) for hydrogen and carbon recoil nuclei for WIMPs with masses of 10 and $100 \mathrm{GeV}$. Only tracks with a length greater than $80 \mathrm{~nm}$ are considered.

The significantly lower density of propane makes it possible to visualize a substantially larger number of tracks of recoil nuclei compared to the number of tracks in a denser emulsion. Since the original WIMP angular distribution is rather wide (see Figures 2-4), the directions of the tracks of the recoil nuclei are correspondingly wide.

Figures 10 and 11 illustrate the fact that WIMPs with small masses $(\sim 10 \mathrm{GeV})$ do not couple effectively with $\mathrm{C}$ and $\mathrm{F}$ nuclei and do not create recoil nuclei tracks with track lengths more than $100 \mathrm{~nm}$ in the octafluoropropane, while such coupling is possible for WIMPs with masses $100 \mathrm{GeV}$. This means that the PICO detector as well as the XENON1T detector would exhibit the best sensitivity to WIMPs with masses $\lesssim 100 \mathrm{GeV}$. 
$\mathrm{C}_{3} \mathrm{~F}_{8}, \mathrm{C} \& \mathrm{~F}$ recoil tracks, WIMP-10,100GeV, Track_length2D $>2 \mathrm{~nm}$

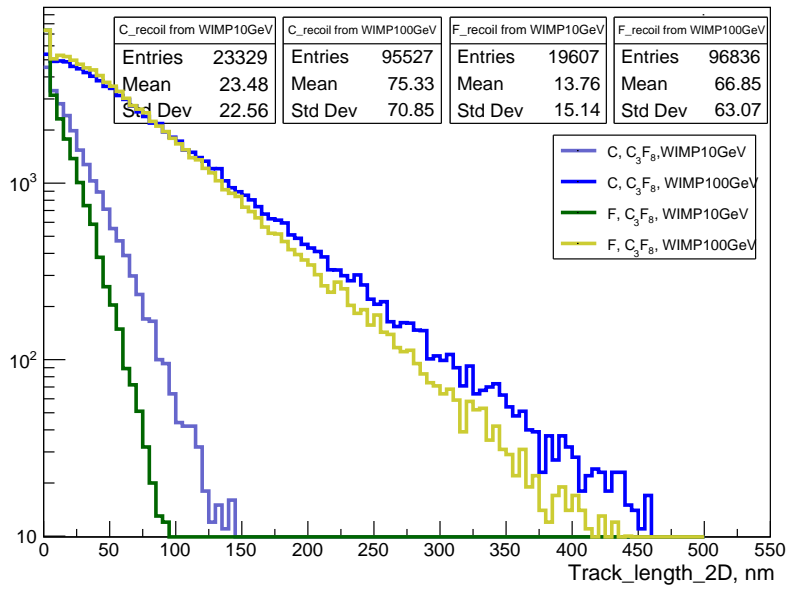

Figure 10. Track length distributions for carbon and fluorine recoil nuclei in $C_{3} F$ for WIMPs with masses 10 and $100 \mathrm{GeV}$.

$\mathrm{C}_{3} \mathrm{~F}_{8}, \Theta_{2 \mathrm{D}}$ for $\mathrm{C}$ \& F recoil tracks, WIMP-10,100GeV, Range $e_{20}>80 \mathrm{~nm}$

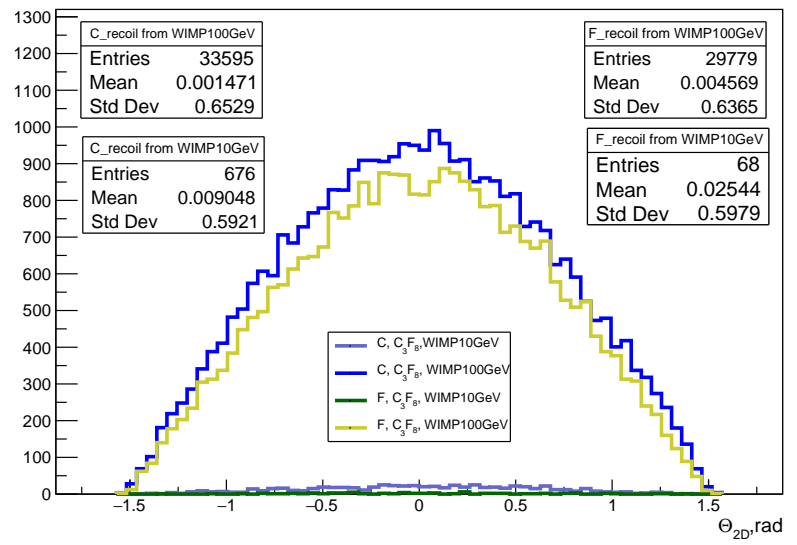

Figure 11. Distributions over the angles between the track and the direction «toward the Cygnus constellation» in $\mathrm{C}_{3} \mathrm{~F}_{8}$ for carbon and fluorine recoil nuclei and WIMP masses 10 and $100 \mathrm{GeV}$.

The angular distribution of recoil nuclei tracks in $\mathrm{C}_{3} \mathrm{~F}_{8}$ has a similar shape as that for both denser (nuclear emulsion) and less dense $\left(\mathrm{C}_{3} \mathrm{H}_{8}\right)$ material. However, the track length cuts $\geq 80 \mathrm{~nm}$ for WIMPs with masses $10 \mathrm{GeV}$ impoverish the statistics of heavier recoil nuclei in a much more significant way.

Calculations of recoil nuclear track lengths and angular distributions from WIMPs with various masses in NIT and UNIT emulsions using SRIM simulations are presented in [22]. An additional modeling scenario, taking into account the crystal structure of the emulsion grains is also considered. Compared to the GEANT4 StandardNR model we used, SRIM simulated tracks, as indicated in [22], are somewhat longer, and the angular distributions of the tracks are wider.

The average values of track length distributions in a standard nuclear emulsion for WIMPs of different masses are given in [23]: $(3.25 \pm 1.73) \cdot 10^{-2} \mu \mathrm{m}$ for $m_{W I M P}=$ $10 \mathrm{GeV} / c^{2}$ and $(9.46 \pm 4.57) \cdot 10^{-2} \mu \mathrm{m}$ for $m_{W I M P}=100 \mathrm{GeV} / c^{2}$. The characteristics of the track length distributions from this work are shown in Figure 8.

\section{Models of Cold Dark Matter (WIMP) in micrOMEGAs. Constraints on WIMP Fluxes for Various Models}

We used the micrOMEGAs 5.2.4 software package [24] - the code for calculating the cold dark matter properties for various supersymmetric extensions of the Standard Modelfor quantitative estimation of the WIMP signal. In the MSSM (Minimal Supersymmetric 
Standard Model) [25] a candidate for the role of a dark matter particle is a light neutralino, which has a spin $1 / 2$ and is a linear combination of superpartners of gauge bosons and Higgs doublet-bino, wino, higgsino1 and higgsino2:

$$
o 1=a \cdot \text { bino }-b \cdot \text { wino }+c \cdot \text { higgsino } 1-d \cdot \text { higgsino } 2
$$

It is now believed that the neutralino in MSSM as a cold dark matter model is almost completely ruled out by experimental constraints [26].

To estimate the cross sections we used the NMSSM (Next-to-Minimal Supersymmetric Standard Model) [27,28] and the IDM (Inert Doublet Model) [29] models for minimal WIMP masses (approximately $10 \mathrm{GeV}$ or less). Higher masses have been ruled out by XENON1T experiment [30].

In the NMSSM a particle with spin $1 / 2$ is assumed as a dark matter particle. Like in the MSSM, it is a linear combination of bino, wino, higgsino1, and higgsino2, but in this case a new superpartner is added-singlino:

$$
o 1=a \cdot \text { bino }-b \cdot \text { wino }+c \cdot \text { higgsino } 1-d \cdot \text { higgsino } 2+e \cdot \operatorname{singlino}
$$

For the NMSSM we considered the SUGRA [31] scenario. For a wide range of model parameters we calculated WIMP masses and values of cross sections of dark matter particle interactions with nucleons (proton and neutron) $\Omega \cdot \mathrm{h}^{2}, \sigma_{p}, \sigma_{n}$. In the "Direct Detection" scenario we also calculated the number of WIMP events per $\mathrm{kg}$ of target per day for hydrogen, carbon, and oxygen targets (see Figure 12).
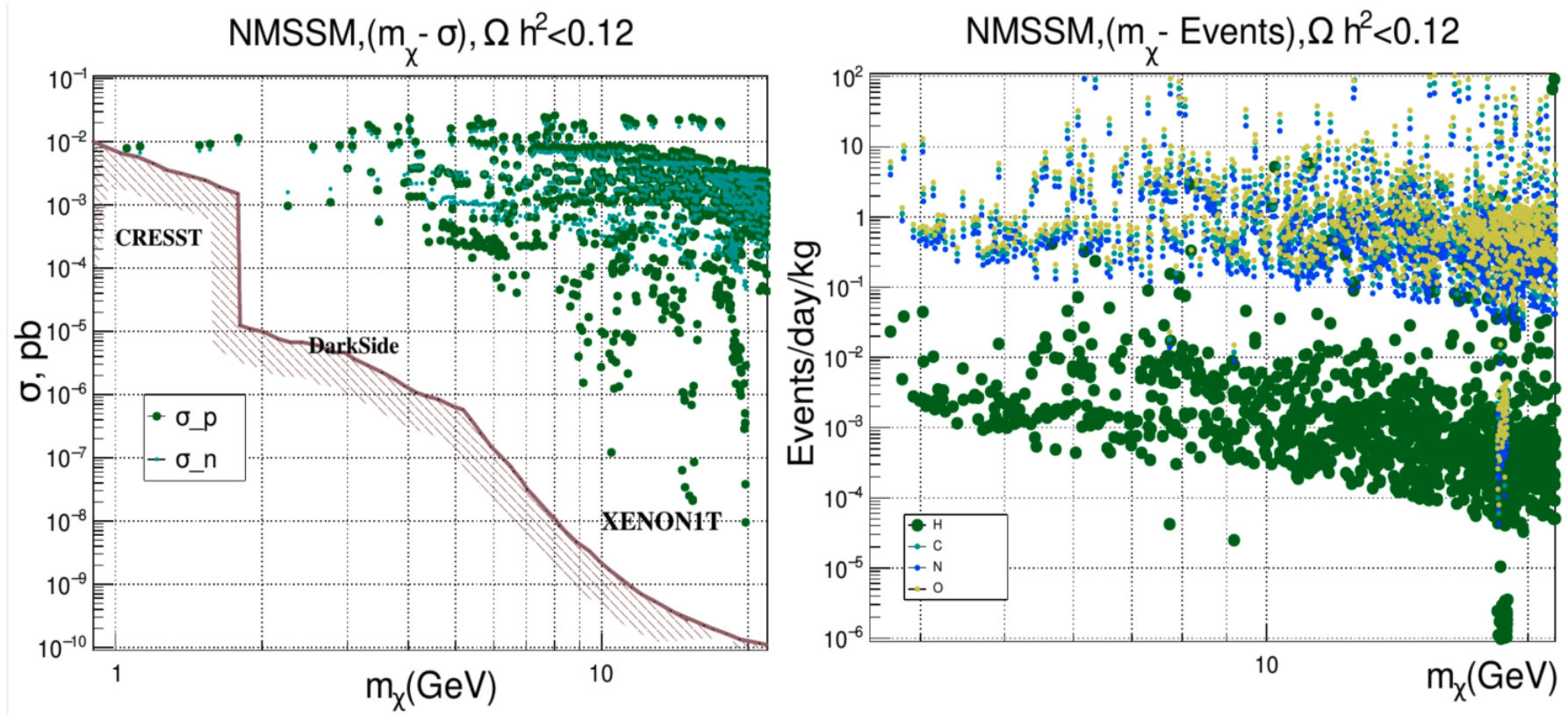

Figure 12. Cross sections of the NMSSM [27] WIMP interactions with nucleons and constraints set by the CRESST [30], DarkSide [32], and XENON1T [33] experiments (left). Number of the NMSSM WIMP events per kg of target (H,C,N,O) per day with the account of the relic density $\Omega \cdot h^{2}$ (right).

The calculations show (see Figure 12) that the NMSSM is also practically ruled out for WIMP masses starting from several GeV by the XENON1T [30] data. DarkSide [32] and CRESST [33] experiments have set the constraints on the WIMP-nucleon interaction cross sections for WIMP masses $\sim 1 \mathrm{GeV}$.

The IDM [29] turned out to be the most promising model in terms of experimental constraints. This model contains, in addition to the Standard Model (SM) particle content, a second complex scalar doublet. The IDM has four new physical states: two charged $\mathrm{H}^{+-}$ and two neutral $H^{0}$ and $A^{0}$, the neutral ones of them can be a cold dark matter particle 
candidate. Here we consider $H^{0}$, the lightest particle of the four, $m_{H^{0}}^{2}<m_{A^{0}}^{2}, m_{H^{ \pm}}^{2}$ as a DM particle candidate. Moreover, in the IDM, a DM particle is a scalar, which means that one does not need to consider the spin-dependent interactions. Thus, the DM interactions with nitrogen and fluorine nuclei could be treated in the same way as the ones with carbon and oxygen nuclei.

Firstly, we need to verify that different IDM options (i.e., different choice of model parameters) do not contradict with the constraints set by the relic density. This comparison is shown in Figure 13.

Figure 14, left shows different IDM models along with existing experimental constraints for different WIMP masses. It can be seen that there are numerous model options which do not contradict the constraints. Figure 14, right shows the IDM simulations in the "WIMP mass-number of events per day per kg of target" parameter space.

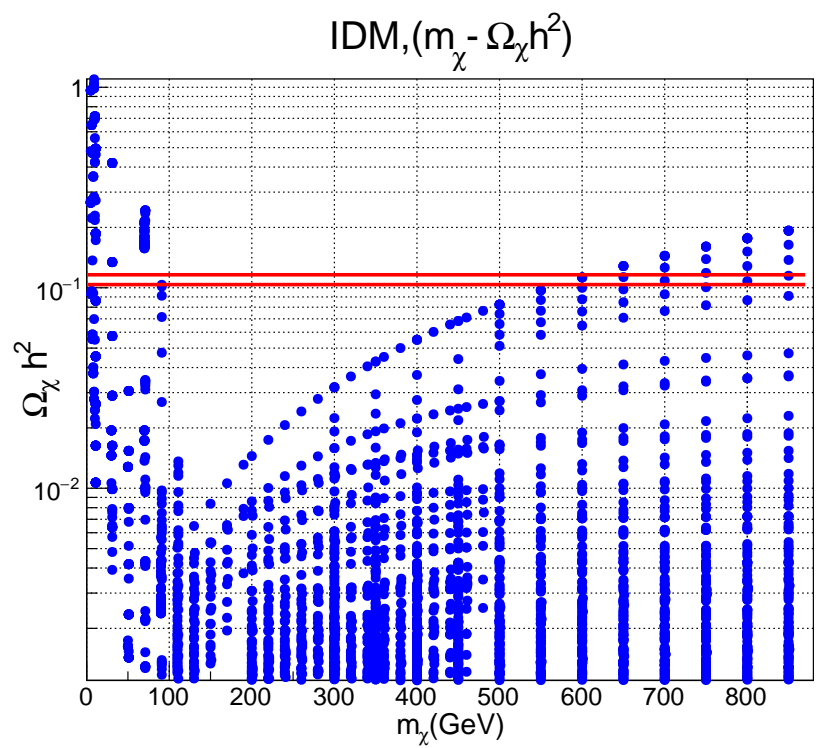

Figure 13. IDM simulations vs. experimental constraints. Blue points denote different IDM variants (for different choice of model parameters) in the "DM mass-relic density" parameter space. Red lines denote the contemporary constraints on the value of $\Omega \cdot h^{2}$.
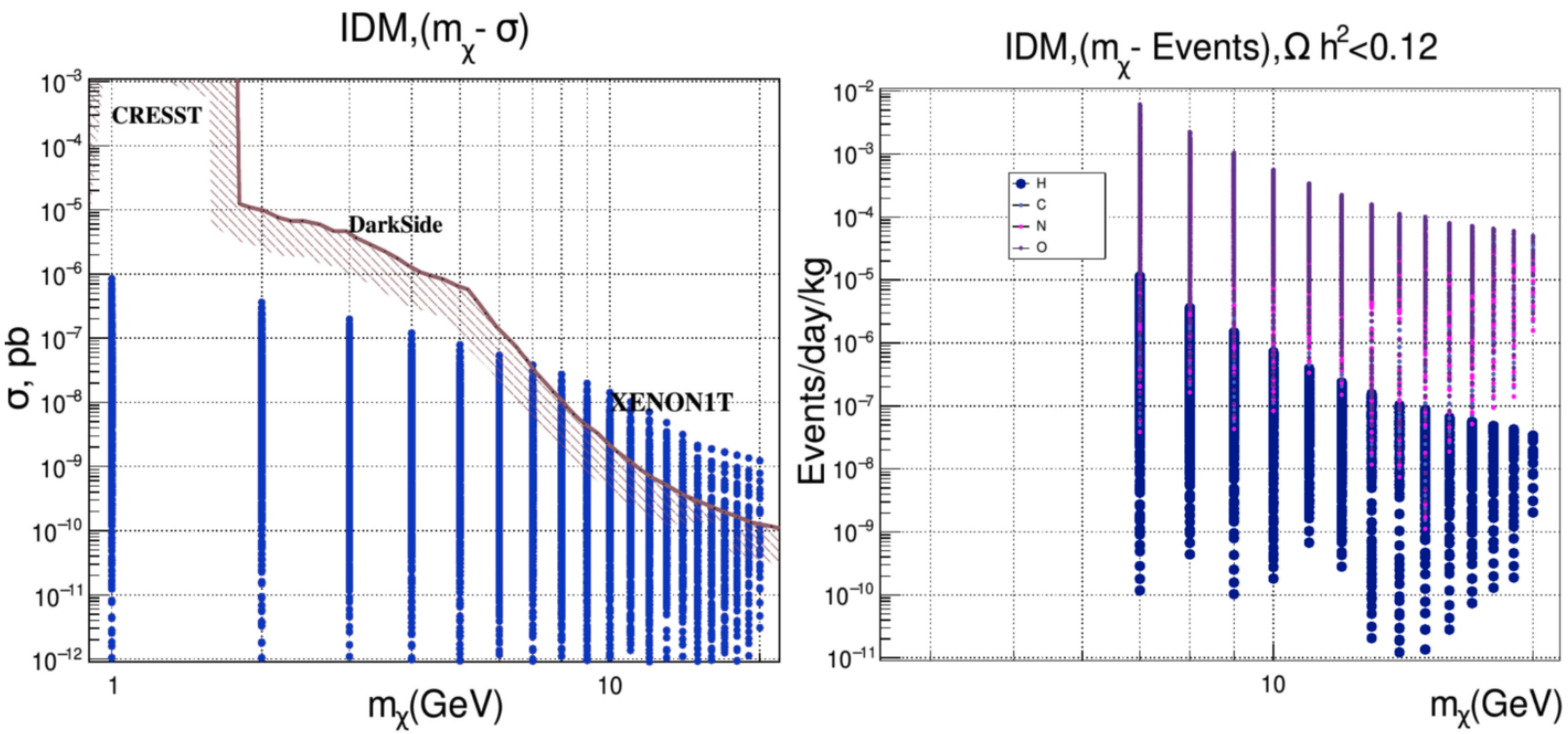

Figure 14. Cross sections for interactions of DM particles of the IDM with nucleons for different masses vs. the existing experimental constraints set by XENON1T [30], DarkSide [32], and CRESST [33] (left). Number of DM-nucleon interactions per kg of target per day for various light targets $(\mathrm{H}, \mathrm{C}, \mathrm{N}, \mathrm{O})$ for different $\mathrm{DM}$ particle masses and $\Omega \cdot \mathrm{h}^{2}<0.12($ right). 
For the numerical estimates we considered WIMPs with mass $10 \mathrm{GeV}$. We also chose the IDM option with the maximal WIMP-nucleon cross section that does not contradict the existing experimental constraints. The corresponding values of WIMP events per day per $\mathrm{kg}$ of target are shown in the conlusive table below.

\section{Elastic Interaction of Solar Neutrinos with Detector Material as a Directional Background for WIMP Observations}

In this work we study the possibility of analyzing the directions and track lengths of recoil nuclei from WIMP-target interactions to search the excess WIMP signal in the direction to the Cygnus constellation. Recoil nuclei from solar neutrino interactions could represent a directional background for such a task.

The expressions for cross sections of neutrino-nucleus elastic scattering used to estimate the neutrino background are presented in, e.g., [34]. The solar neutrino energies were modeled after the corresponding spectrum [35] starting from the energy $0.5 \mathrm{MeV}$.

Figure 15 shows the distributions of the recoil nuclei from solar neutrino interactions in denser (nuclear emulsion) and less dense $\left(\mathrm{C}_{3} \mathrm{H}_{8}\right.$ and $\left.\mathrm{C}_{3} \mathrm{~F}_{8}\right)$ material in the "neutrino energy-recoil nucleus track length" parameter space. It is evident from this figure that hydrogen recoil nuclei from elastic scattering of neutrinos with energies $E>0.8 \mathrm{MeV}$ can form tracks with track lengths more than $2 \mathrm{~nm}$. For carbon nuclei the same is true for neutrinos with energies $E>3 \mathrm{MeV}$. Thus, the contribution from the carbon recoil nuclei is expected to be minimal. A characteristic congestion of dots in the solar neutrino energy region $(\sim 1-2) \mathrm{MeV})$ is due to the form of the intrinsic neutrino spectrum.

Figure 16 shows the distributions of the recoil nuclei from solar neutrinos in $\mathrm{C}_{3} \mathrm{H}_{8}$, $\mathrm{C}_{3} \mathrm{~F}_{8}$, and nuclear emulsion over track lengths. If one applies cuts on track lengths according to the sensitivity of nanoemulsions to the directions of recoil nuclei tracks ( $\geq 60-80 \mathrm{~nm})$, it becomes evident that the main contribution to the directional solar neutrino signal would be from hydrogen nuclei.

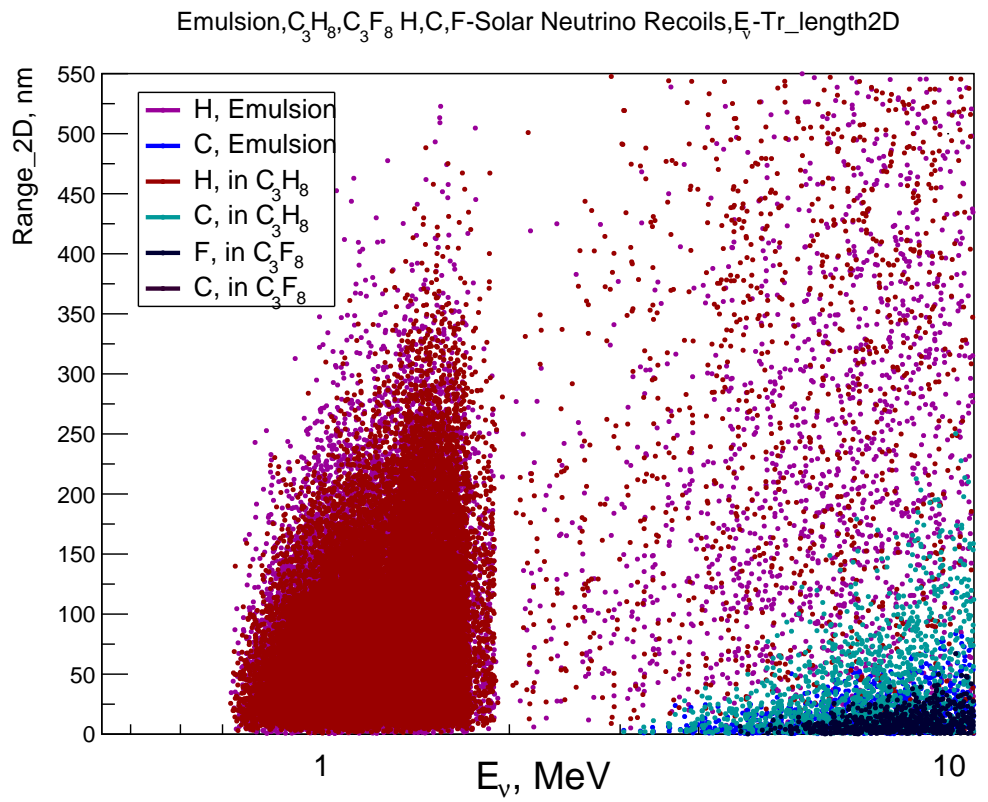

Figure 15. Distributions of the recoil nuclei from solar neutrino interactions over neutrino energies and recoil nuclei track lengths. The points represent simulated hydrogen $(\mathrm{H})$ and carbon $(\mathrm{C})$ recoil nuclei events in nuclear emulsion and propane. A cut (minimal track length $\sim 2 \mathrm{~nm}$ ) has been applied to all the simulated tracks. This cut is motivated by GEANT4 capabilities. 
Emulsion, $\mathrm{C}_{3} \mathrm{H}_{8}, \mathrm{C}_{3} \mathrm{~F}_{8}, \mathrm{H}, \mathrm{C}, \mathrm{F}$ - Solar Neutrino Recoils,Track_length2D $>2 \mathrm{~nm}$

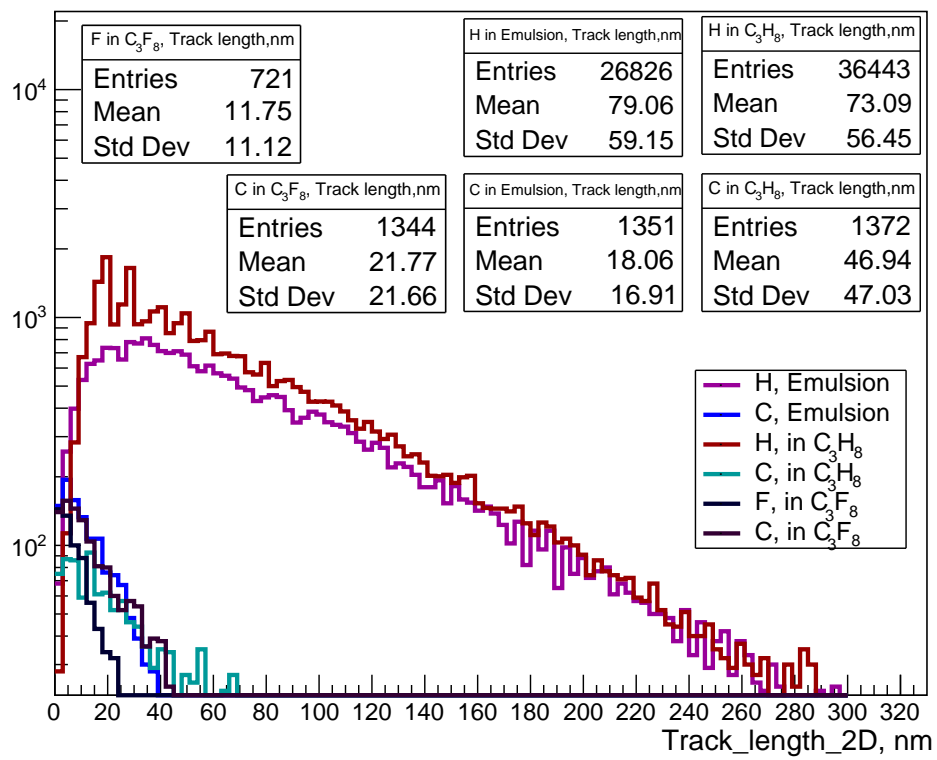

Figure 16. Distribution of track lengths of hydrogen $(\mathrm{H})$ and carbon $(\mathrm{C})$ recoil nuclei from solar neutrino interactions in nuclear emulsion, $\mathrm{C}_{3} \mathrm{H}_{8}$ and $\mathrm{C}_{3} \mathrm{~F}_{8}$.

\section{Discussion and Conclusions}

In this work we estimated the annual signal from a WIMP with a mass of $10 \mathrm{GeV}$ in $30 \mathrm{~kg}$ of nuclear emulsion, $500 \mathrm{l}$ of liquid $\mathrm{C}_{3} \mathrm{H}_{8}$, and $500 \mathrm{~L}$ of liquid $\mathrm{C}_{3} \mathrm{~F}_{8}$. It was found that approximately 1 event per year is expected in the considered sensitive volume of the emulsion. Accordingly, in order to register approximately 10 events related to WIMPs, it is necessary to expose and scan approximately $300 \mathrm{~kg}$ of emulsion, which requires significant material and human resources. In targets with a lower density, $\mathrm{C}_{3} \mathrm{H}_{8}$ and $\mathrm{C}_{3} \mathrm{~F}_{8}$, entirely consisting of light nuclei, and also providing long track lengths of nuclei, there will be 24 and 194 events from WIMPs per year.

These estimates allow us to conclude that, taking into account the existing experimental constraints on the elastic interaction cross sections of the WIMP-nucleus interactions, it makes sense in the direct detection scenario to consider lighter and less dense targets as a sensitive element of the detector system.

These results, as well as estimates of recoil events number from solar neutrinos, are presented in the table (see Figure 17).

\begin{tabular}{|c|c|c|c|c|c|}
\hline $\begin{array}{c}\text { detector } \\
\text { substance, } \\
\text { mass or volume }\end{array}$ & $\begin{array}{c}\text { Element, } \\
\text { (\% in } \\
\text { substance) }\end{array}$ & $\begin{array}{c}\mathrm{N}_{\text {wimp }} \text { per day } \\
\text { in 1 kg of } \\
\text { an element }\end{array}$ & $\begin{array}{c}\mathrm{N}_{\text {wimp }} \\
\text { per year } \\
\text { in 1 kg of } \\
\text { substance }\end{array}$ & $\begin{array}{c}\mathrm{N}_{\text {wimp }} \\
\text { per year }\end{array}$ & $\begin{array}{c}\mathrm{N}_{\mathrm{v}} \\
\text { per year }\end{array}$ \\
\hline emulsion, 30 kg & $\mathrm{H}(1.6)$ & $7.03^{*} 10^{-7}$ & & & \\
\hline emulsion, 30 kg & $\mathrm{C}(10.1)$ & $3.23^{*} 10^{-4}$ & $3.14^{*} 10^{-2}$ & 0.94 & $<2.51^{*} 10^{-2}$ \\
\hline emulsion, 30 kg & $\mathrm{N}(2.7)$ & $4.32^{*} 10^{-4}$ & & & \\
\hline emulsion, 30 kg & $\mathrm{O}(7.4)$ & $5.51^{*} 10^{-4}$ & & & \\
\hline C3H8, 500 l & $\mathrm{H}(18.2)$ & $7.03^{*} 10^{-7}$ & $4.82^{*} 10^{-2}$ & 23.75 & $<2.32^{*} 10^{-2}$ \\
\hline C3H8, 500 l & $\mathrm{C}(81.8)$ & $3.23^{*} 10^{-4}$ & & & $<4.47^{*} 10^{-2}$ \\
\hline C3F8, 500 l & $\mathrm{C}(19.1)$ & $3.23^{*} 10^{-4}$ & $2.42^{*} 10^{-1}$ & 194.0 & \\
\hline C3F8, 500 l & $\mathrm{F}(80.9)$ & $7.44 * 10^{-4}$ & & & \\
\hline
\end{tabular}

Figure 17. Estimates of number of WIMP and neutrino events in the emulsion, $\mathrm{C}_{3} \mathrm{H}_{8}$, and $\mathrm{C}_{3} \mathrm{~F}_{8}$. 
Author Contributions: Conceptualization, A.A. and E.U.; methodology, E.U.; software, A.A.; validation, T.R.; formal analysis, I.V. and V.G.; investigation, E.K. (Emil Khalikov); resources, E.K. (Evgeny Kurochkin); data curation, I.V.; writing—original draft preparation, E.U.; writing-review and editing, A.A.; visualization, V.G.; supervision, T.R.; project administration, T.R.; funding acquisition, A.A. All authors have read and agreed to the published version of the manuscript.

Funding: This research was funded by Basis Foundation grant number \#20-2-9-26-1 and by the Interdisciplinary Scientific and Educational School of Moscow University "Fundamental and Applied Space Research".

Institutional Review Board Statement: Not applicable.

Informed Consent Statement: Not applicable.

Acknowledgments: We express our gratitude to A. Pukhov for advice on some theoretical issues.

Conflicts of Interest: The authors declare no conflict of interest. The funders had no role in the design of the study; in the collection, analyses, or interpretation of data; in the writing of the manuscript, or in the decision to publish the results.

\section{References}

1. Milgrom, M. A modification of the Newtonian dynamics as a possible alternative to the hidden mass hypothesis. Astrophys. J. 1983, 270, 365-370. [CrossRef]

2. Toky, H.R.; Carignan, C. Galaxy mass models: MOND versus dark matter haloes. Mon. Not. R. Astron. Soc. 2014, 439, 2132-2145.

3. Carroll, S.M.; Remmen, G.N. What is the entropy in entropic gravity? Phys. Rev. D 2016, 93, 124052. [CrossRef]

4. Haug, E.G. Can the Standard Model Predict a Minimum Acceleration That Gets Rid of Dark Matter? J. High Energy Phys. Gravit. Cosmol. 2021, 7, 602. [CrossRef]

5. Steigman, G.; Turner, M.S. Cosmological constraints on the properties of weakly interacting massive particles. Nucl. Phys. B 1985, 253, 375-386. [CrossRef]

6. Abazajian, K.N.; Acero, M.A.; Agarwalla, S.K.; Aguilar-Arevalo, A.A.; Albright, C.H.; Antusch, S.; Arguelles, C.A.; Balantekin, A.B.; Barenboim, G.; Barger, V.; et al. Light Sterile Neutrinos: A White Paper. arXiv 2012, arXiv:1204.5379.

7. Giomataris, Y.; Rebourgeard, P.; Robert, J.P.; Charpak, G. MICROMEGAS: A high-granularity position-sensitive gaseous detector for high particle-flux environments. Nucl. Instrum. Methods Phys. Res. Sect. A Accel. Spectrometers Detect. Assoc. Equip. 1996, 376, 29-35. [CrossRef]

8. Wang, S.Y. Graphene-based detectors for directional dark matter detection. Eur. Phys. J. C 2019, 79, 561. [CrossRef]

9. Green, A.M. WIMP direct detection and halo structure. In Symposium-International Astronomical Union; Cambridge University Press: Cambridge, UK, 2004; Volume 220.

10. Belanger, G.; Boudjema, F.; Pukhov, A. micrOMEGAs: A code for the calculation of Dark Matter properties in generic models of particle interaction. arXiv 2014, arXiv:1402.0787.

11. Aleksandrov, A.; Agafonova, N.; Anokhina, A.; Asada, T.; Ashikhmin, V.V.; Bodnarchuk, I.; Buonaura, A.; Chernyavskii, M.; Chukanov, A.; D'Ambrosio, N.; et al. [NEWSdm Collaboration] Discovery potential for directional Dark Matter detection with nuclear emulsions. Eur. Phys. J. C 2018, 78, 758. [CrossRef]

12. Asada, T.; Naka, T.; Kuwabara, K.I.; Yoshimoto, M. The development of a super-fine-grained nuclear emulsion. Prog. Theor. Exp. Phys. 2017, 2017, 063H01. [CrossRef]

13. Brugnera, R.; Guler, A.M.; Di Marco, N.; Agafonova, N.; Patrizii, L.; Naganawa, N.; Shibuya, H.; Vuilleumier, J.L.; Ustyuzhanin, A.; Shchedrina, T.; et al. Final Results of the OPERA Experiment on $v \tau$ Appearance in the CNGS Neutrino Beam. Phys. Rev. Lett. 2018, 120, 211801.

14. Amole, C.; Ardid, M.; Arnquist, I.J.; Asner, D.M.; Baxter, D.; Behnke, E.; Bressler, M.; Broerman, B.; Cao, G.; Chen, C.J.; et al. Dark matter search results from the complete exposure of the PICO-60 C 3 F 8 bubble chamber. Phys. Rev. D 2019, $100,022001$. [CrossRef]

15. Lewin, J.D.; Smith, P.F. Review of mathematics, numerical factors, and corrections for dark matter experiments based on elastic nuclear recoil. Astropart. Phys. 1996, 6, 87-112. [CrossRef]

16. Alenazi, M.S.; Gondolo, P. Directional recoil rates for WIMP direct detection. Phys. Rev. D 2008, 77, 043532. [CrossRef]

17. Smith, P.F.; Lewin, J.D. Dark matter detection. Phys. Rep. 1990, 187, 203-280. [CrossRef]

18. Savage C.; Gondolo P.; Freese K. Can WIMP spin dependent couplings explain DAMA data, in light of null results from other experiments? Phys. Rev. D 2004, 70, 123513. [CrossRef]

19. Engel, J. Nuclear form factors for the scattering of weakly interacting massive particles. Phys. Lett. B 1991, 264, 114-119. [CrossRef]

20. Ellis, J.; Flores, R.A. Elastic supersymmetric relic-nucleus scattering revisited. Phys. Let. B 1991, 263, 259-266. [CrossRef]

21. Book For Application Developers. Available online: https://geant4-userdoc.web.cern.ch/UsersGuides/ForApplicationDeveloper/ $\mathrm{html} /$ index.html (accessed on 1 May 2021). 
22. Alexandrov, A.; De Lellis, G.; Di Crescenzo, A.; Golovatiuk, A.; Tioukov, V. Tioukov Directionality preservation of nuclear recoils in an emulsion detector for directional dark matter search. J. Cosmol. Astropart. Phys. 2021, 2021, 047. [CrossRef]

23. Couturier, C.; Zopounidis, J.P.; Sauzet, N.; Naraghi, F.; Santos, D. Dark Matter directional detection: Comparison of the track direction determination. J. Cosmol. Astropart. Phys. 2017, 2017, 027. [CrossRef]

24. Barducci, D.; Bélanger, G.; Boudjema, F.; Da Silva, J.; Goudelis, A.; Kraml, S.; Laa, U.; Pukhov, A.; Semenov, A.; Zaldivar B. micrOMEGAs: The User's Manual, Version 5.0. 2021. Available online: https://lapth.cnrs.fr/micromegas/(accessed on 1 May 2021).

25. Bottino, A.; Fornengo, N.; Scopel, S. Light Relic Neutralino. Phys. Rev. D 2003, 67, 063519. [CrossRef]

26. Han, T.; Liu, Z.; Su, S. Light neutralino dark matter: Direct/indirect detection and collider searches. J. High Energy Phys. 2014, 2014, 093. [CrossRef]

27. Ellwanger, U.; Hugonie, C. NMSPEC: A Fortran code for the sparticle and Higgs masses in the NMSSM with GUT scale boundary conditions. Comput. Phys. Commun. 2007, 177, 399-407. [CrossRef]

28. Belanger, G.; Boudjema, F.; Hugonie, C.; Pukhov, A.; Semenov, A. Relic density of dark matter in the next-to-minimal supersymmetric standard model. J. Cosmol. Astropart. Phys. 2005, 2005, 001. [CrossRef]

29. Gustafsson, M. The inert doublet model and its phenomenology. arXiv 2011, arXiv:1106.1719.

30. Aprile, E.; Aalbers, J.; Agostini, F.; Alfonsi, M.; Amaro, F.D.; Anthony, M.; Arneodo, F.; Barrow, P.; Baudis, L.; Bauermeister, B.; et al. First dark matter search results from the XENON1T experiment. Phys. Rev. Lett. 2017, 119, 181301. [CrossRef]

31. Chakravarty, G.K.; Gupta, G.; Lambiase, G.; Mohanty, S. Plateau inflation in SUGRA-MSSM. Phys. Lett. B 2016, 760, $263-266$.

32. Agnes, P.; Albuquerque, I.F.M.; Alexander, T.; Alton, A.K.; Araujo, G.R.; Asner, D.M.; Ave, M.; Back, H.O.; Baldin, B.; Batignani, G.; et al. Low-mass dark matter search with the DarkSide-50 experiment. Phys. Rev. Lett. 2018, 121, 081307. [CrossRef]

33. Abdelhameed, A.H.; Angloher, G.; Bauer, P.; Bento, A.; Bertoldo, E.; Bucci, C.; Canonica, L.; D'Addabbo, A.; Defay, X.; Di Lorenzo, S.; et al. First results from the CRESST-III low-mass dark matter program. Phys. Rev. D 2019, 100, 102002. [CrossRef]

34. O'Hare, C.A.; Green, A.M.; Billard, J.; Figueroa-Feliciano, E.; Strigari, L.E. Readout strategies for directional dark matter detection beyond the neutrino background. Phys. Rev. D Part. Fields Gravit. Cosmol. 2015, 92, 063518. [CrossRef]

35. Haxton, W.C.; Hamish Robertson, R.G.; Serenelli, A.M. Solar Neutrinos: Status and Prospects. Ann. Rev. Astron. Astrophys. 2013, 51, 21-61. [CrossRef] 